\section{Rabdomiólisis aguda asociada a inhalación de cocaína}

\section{Sr. Director:}

La cocaína es una droga de abuso cuyo consumo se está extendiendo en nuestro medio. Su empleo desencadena múltiples complicaciones médicas que pueden comprometer la vida. El diagnóstico etiológico de dichos eventos y la instauración precoz de una terapia adecuada son fundamentales para prevenir la progresión de la gravedad de estos cuadros. Presentamos el caso de un paciente varón de 29 años de edad, sin antecedentes médicos de interés, que 12 horas tras la inhalación de cocaína (crack) es hallado estuporoso en su domicilio. Es trasladado al hospital, donde llegó consciente con desorientación temporo-espacial y estable hemodinámicamente. En la exploración física destacan miembros inferiores (MMII) empastados y calientes desde la raíz de los mismos. Los pulsos distales y la sensibilidad estaban preservados. Analíticamente se objetivó un fracaso renal oligoanúrico (urea: $50 \mathrm{mg} / \mathrm{dl}$; creatinina: $5,2 \mathrm{mg} / \mathrm{dl}$; Ccr: 46,71 $\mathrm{ml} / \mathrm{min}$ ), rabdomiólisis (CPK máx: $299.430 \mathrm{U} / \mathrm{ml}$ ), hiponatremia (Na: $133 \mathrm{mEq} / \mathrm{l})$, hiperpotasemia (K: 7,4 mEq/l) y acidosis metabólica (lactato: $5 \mathrm{mg} / \mathrm{dl}$ ). La tomografía axial computarizada (TAC) craneal no mostró hallazgos patológicos. La TAC abdominal evidenció un aumento de tamaño de ambas siluetas renales y distensión gluteofemoral, compatible con un proceso de licuefacción muscular. Ingresa en la Unidad de Medicina Intensiva, instaurándose hemodiafiltración veno-venosa continua, fluidoterapia y alcalinización urinaria. A las 48 horas de su ingreso el enfermo manifestó un deterioro progresivo de la sensibilidad en MMII. Ante el desarrollo de un síndrome compartimental agudo se decidió hacer fasciotomías laterales en ambos muslos. El paciente permaneció hemodinámicamente estable, intubado y conectado a ventilación mecánica. Al cuarto día se le extubó y al noveno día fue dado de alta al Servicio de Cirugía Plástica. En el momento del alta el enfermo seguía requiriendo hemodiálisis intermitente (urea: $165 \mathrm{mg} / \mathrm{dl}$; creatinina: $5 \mathrm{mg} / \mathrm{dl}$; Ccr: $26,68 \mathrm{ml} / \mathrm{min}$ ) y la CPK sérica se había normalizado.

La rabdomiólisis es un síndrome clínico caracterizado por la necrosis con citolisis del músculo esquelético y liberación de las enzimas musculares al plasma, siendo las cifras de CPK sérica las que se emplean para establecer el diagnóstico por su mayor sensibilidad ${ }^{1}$. El espectro de gravedad de este cuadro oscila desde la elevación asintomática de enzimas musculares hasta situaciones de riesgo vital, con compromiso de la musculatura respiratoria, hiperpotasemia y/o fracaso renal agudo ${ }^{2}$. Además de los antecedentes traumáticos se han descrito como agentes desencadenantes de rabdomiólisis consumo de drogas (heroína, anfetaminas intravenosas, marihuana y cocaína), deficiencias enzimáticas, miopatías, trastornos hidroelectrolíticos, infecciones, trastornos inflamatorios, etc. ${ }^{1-5}$.

El manejo de los pacientes con rabdomiólisis está dirigido a evitar nuevas complicaciones, previniendo y tratando el fracaso renal agudo durante la fase de mioglobinuria. Las medidas básicas son la hidratación, la alcalinización de la orina con bicarbonato sódico y el empleo de diuréticos como el manitol o la furosemida ${ }^{2}$.

E. HERNÁNDEZ MEDINA, J.M. MUÑOZ UNAMUNO,

A. VILLANUEVA ORTIZ Y M. SÁNCHEZ PALACIOS

Servicio de Medicina Intensiva. Hospital Insular de Las Palmas de Gran Canaria.

\section{BIBLIOGRAFÍA}

1. Brody SL, Wrenn KD, Wilber MM, Slovis CM. Predicting the severity of cocaine-associated rhabdomyolysis. Ann Emerg Med. 1990;19:1137-43.

2. El-Hayek BM, Nogue S, Alonso D, Poch E. Rhabdomyolysis, compartment syndrome and acute kidney failure related to cocaine consume. Nefrología. 2003;23:469-70.

3. Shanti CM, Lucas CE. Cocaine and the critical care challenge. Crit Care Med. 2003;31:1851-9.

4. Herzlich BC, Arsura EL, Pagala M, Grob D. Rhabdomyolysis related to cocaine abuse. Ann Intern Med. 1988;109:335-6.

5. Pogue VA, Nurse HM. Cocaine-associated acute myoglobinuric renal failure. Am J Med. 1989;86:183-6. 